\title{
Repositório para o rastreamento de requisitos funcionais derivados de regras de negócio
}

\author{
Adriana Cristina de Melo ${ }^{1}$ \\ Paulo Sérgio Muniz Silva ${ }^{2}$
}

\begin{abstract}
Resumo: As mudanças nos negócios de uma empresa levam à adaptação das regras de negócio e dos sistemas de informação. Mas antes de executar as mudanças deve-se identificar o que precisa ser alterado no sistema e estimar o tempo e o custo para efetuar a manutenção. A rastreabilidade de requisitos auxilia a análise das consequências das mudanças e a verificação de inconsistências entre os requisitos. Porém, nos sistemas em que as regras de negócio e os requisitos são implementados juntos dificulta-se a rastreabilidade, pois as regras ficam escondidas nos requisitos e os detalhes de implementação são conhecidos apenas pelos seus desenvolvedores. Este trabalho propõe um repositório de requisitos de software, restrito aos funcionais, que permite relacionar as decisões e as regras de negócio e os requisitos de software, possibilitando rastrear os requisitos e sua origem nos negócios. Com a história dos requisitos documentada, incluindo as definições de negócio, é possível atribuir responsabilidades e efetuar avaliações de modificações futuras e de desenvolvimento de novos sistemas. A partir de um protótipo ilustra-se a aplicabilidade do repositório proposto nas áreas de negócio e de sistema.
\end{abstract}

Palavras-chave: Rastreabilidade de requisitos. Regras de negócio. Requisitos funcionais.

\begin{abstract}
Changes in a company's business lead to an adaptation of business rules and information systems. But before performing these changes one must identify what needs to be modified in the system and estimate the time and cost for performing maintenance. Requirements traceability assists the analysis of consequences of changes and the verification of inconsistencies between requirements. However, systems where business rules and requirements are implemented together hampers traceability, because the business rules remain hidden behind the requirements and implementation details are known only by their developers. This work proposes a software requirements repository, restricted to the functional ones, which enables the correlation of business decisions, business rules and software requirements, allowing requirements traceability to its origins in business. With the history of requirements documented, including the business definitions, it is possible to assign responsibilities and undertake evaluations of future modifications and development of new systems. From a prototype it is illustrated the applicability of the proposed repository in the business and system areas.
\end{abstract}

Keywords: Business rules. Functional requirements. Requirements traceability.

\section{Introdução}

As mudanças nos negócios de uma empresa são causadas por decisões internas ou externas, tais como as relacionadas a governo, leis, regulamentos, e levam à adaptação das regras de negócio e dos sistemas de informação.

\footnotetext{
${ }^{1}$ Instituto de Pesquisas Tecnológicas do Estado de São Paulo - São Paulo- SP - Brasil.

\{adrianameipt.br\}

${ }^{2}$ Instituto de Pesquisas Tecnológicas do Estado de São Paulo - São Paulo -SP - Brasil / Escola Politécnica da Universidade de São Paulo - São Paulo-SP - Brasil.

\{paulo.muniz@usp.br\}
}

http://dx.doi.org/10.5335/rbca.2014.3787 
Um processo de manutenção de sistema pode afetar o custo, o cronograma e a qualidade de um projeto, devido a alterações desnecessárias, geração de retrabalho, causando a insatisfação do cliente, podendo até levar ao fracasso do projeto. Por essas razões, antes de executar as mudanças deve-se identificar o que precisa ser alterado no sistema e estimar o tempo e o custo para efetuar a manutenção.

A rastreabilidade de requisitos auxilia a análise das consequências das mudanças e a verificação de inconsistências entre os requisitos por sua capacidade de descobrir a história de cada característica do sistema [1]. Porém, nos sistemas em que as regras de negócio e os requisitos são implementados juntos dificulta-se a rastreabilidade, pois as regras ficam escondidas nos requisitos e os detalhes de implementação são conhecidos apenas pelos seus desenvolvedores. Para evitar esse problema, as regras de negócio e os requisitos devem ser documentados em separado para constituírem itens que possam ser explicitamente rastreados.

A primeira etapa para essa documentação é estabelecer a diferença entre regras de negócio e requisitos. Segundo Ross [2], as regras de negócio representam o funcionamento do negócio, enquanto os requisitos representam as necessidades que devem ser atendidas pelo sistema, ou seja, os requisitos são derivados das regras de negócio. A segunda etapa é determinar um modo de documentar as regras de negócio e os requisitos de software separadamente. Com isso, define-se uma estratégia de rastreabilidade que pode então ser apoiada por um repositório que permita rastrear os requisitos (restrito aos funcionais e representados por casos de uso) e sua origem nos negócios. A seção 2 deste artigo descreve conceitos de regra de negócio e de requisito de software para diferenciá-los. Além disso, a seção mostra conceitos de caso de uso e sua relação com a regra de negócio. A seção 3 apresenta os conceitos e metamodelos de rastreabilidade de requisitos. Na seção 4 é mencionado o modelo conceitual do repositório de requisitos funcionais de software e regras de negócio, e na seção 5, tem-se um exemplo de aplicação do repositório. A seção 6 mostra uma avaliação preliminar da aplicação do repositório. A seção 7 apresenta as conclusões.

\section{Regra de negócio e requisito de software}

Para que os sistemas sejam consistentes com os negócios, deve haver uma documentação, desde a origem nos negócios até a implementação nos sistemas, que possibilite determinar as implicações das mudanças. Porém, as regras de negócio e os requisitos de software são conceitos diferentes e precisam ser documentados separadamente para possibilitar rastreá-los.

\subsection{Regra de negócio}

Segundo Business Rule Group [3], regra de negócio é uma declaração que define ou restringe características do negócio. Destina-se a estruturar, controlar ou influenciar o funcionamento do negócio.

Para obter as regras de negócio, é preciso explorar todo o negócio, coletar cada declaração que descreve como as empresas fazem os negócios hoje e antecipar as necessidades do negócio de amanhã [4]. Além de estarem de acordo com o negócio, as regras não devem ser codificadas em aplicações, pois não pertencem ao sistema de informação. Essas são definidas/gerenciadas pela empresa e podem ser usadas para ajudar a manter o sistema de informação, refletindo os negócios da empresa [5].

Conforme Steinke e Nickolette [6], as regras de negócio devem ser documentadas para que possam ser usadas no levantamento de requisitos, pois quando uma regra de negócio é desconhecida do desenvolvedor o sistema pode não funcionar como esperado. Nesse caso, um repositório de regras de negócio pode ajudar na consulta às regras e na sua relação com os requisitos antes do início do desenvolvimento. Depois de armazenadas, desde que não haja alterações, as regras não precisam ser levantadas e validadas a cada projeto.

De acordo com Nijpels [7], as regras de negócio são parte do processo de análise de requisitos. Para ele, a combinação da modelagem de processos de negócio, gestão de regras de negócio e modelagem conceitual de dados, descreve o modelo de negócio que sustenta os requisitos de software.

\subsection{Requisito de software}

Requisito de software é uma característica desenvolvida ou adaptada em um software para resolver um problema. Esse problema pode ser automatizar uma tarefa, apoiar processos de negócio da empresa, corrigir 
falhas existentes no software ou controlar um dispositivo. Os requisitos de software são uma combinação de requisitos, pessoas de diferentes níveis da empresa e do ambiente em que o software irá operar [8].

De acordo com Gottesdiener [9], requisito é definido como a capacidade operacional de um sistema ou de um processo para satisfazer as necessidades do usuário. Os requisitos são classificados em funcionais (funcionalidades esperadas pelo usuário) e não funcionais (atributos de qualidade do software como desempenho e segurança).

Para Wiegers [10], um consenso na área de software é que os requisitos devem ser documentados. A documentação que contém a definição do software é chamada especificação de requisitos. Essa especificação pode estar em um documento, em um banco de dados, em um caso de uso, em um repositório de requisitos, ou em uma combinação desses tipos.

Segundo Pressman [11], à medida em que os requisitos são coletados, as funções e as características do sistema começam a se materializar. No entanto, para avançar nas atividades de engenharia de software, a equipe de desenvolvimento tem que entender como essas funções e características serão utilizadas pelos usuários. Para isso, desenvolvedores e usuários criam um conjunto de cenários que identificam o uso do sistema a ser desenvolvido. Os cenários, que podem ser representados por casos de uso, fornecem uma descrição de como o sistema será usado.

\subsection{Caso de uso}

Para representar os requisitos funcionais e suas especificações utiliza-se o caso de uso [12], um modelo bastante difundido na comunidade de engenharia de software. Para Fowler [13], os casos de uso são uma ferramenta que ajuda no entendimento dos requisitos funcionais, pois descrevem as interações entre os usuários e o sistema, fornecendo uma narrativa da utilização do sistema. Em outras palavras, os casos de uso modelam o comportamento do sistema do ponto de vista de seus usuários. Para representar os casos de uso existem vários formatos de gabaritos. O gabarito usado neste trabalho é o proposto pelo Rational Unified Process [14] que dispõe do seguinte formato:

- nome: nome do caso de uso;

- descrição: descreve a finalidade do caso de uso;

- ator: pode ser uma pessoa, uma empresa ou um sistema que chama o sistema para executar um serviço. Geralmente, o caso de uso é iniciado por um ator;

- fluxo de eventos: descrição do que o sistema deve fazer e não como deve ser feito. Ele é formado pelo fluxo básico e pelo fluxo alternativo;

- fluxo básico: é formado por um conjunto de passos de ação dos atores para alcançar o objetivo, seja um ator realizando uma tarefa ou passando uma informação para outro ator;

- fluxo alternativo: cada ponto no qual o comportamento desvia para uma condição particular, podendo ser uma condição de falha ou de sucesso alternativo;

- precondição: descreve a condição que deve ser verdadeira antes que o caso de uso comece;

- pós-condição: descreve a condição que o sistema deve estar no final do caso de uso;

- requisitos especiais: descreve os requisitos não funcionais relacionados ao caso de uso;

- pontos de extensão: define a localização dos pontos de extensão no fluxo de eventos. Esse tem um nome e uma lista de referências a um ou mais locais do fluxo de eventos, sendo referência para os demais casos de uso que deverão ser executados.

Para Cockburn [15], os casos de uso são como uma estrutura de ligação do projeto. Tendo essa estrutura, os casos de uso proporcionam o rastreamento dos requisitos. Se um novo caso de uso é adicionado, nota-se que o escopo aumentou e, portanto, o plano de projeto deve mudar [16]. 


\subsection{Caso de uso e regra de negócio}

Kulak e Guiney [16] propõem uma combinação de casos de uso com as regras de negócio, na qual os casos de uso tratam os requisitos por meio das interações dos atores, e o sistema e as regras de negócio regem essas interações, estabelecendo o ambiente em que o sistema opera. O relacionamento do caso de uso com a regra de negócio é útil, pois quando ocorre uma mudança na regra de negócio identificam-se os casos de uso que precisam ser verificados e vice-versa. Deve haver uma sinergia do caso de uso com a regra para que o sistema atenda às necessidades de negócio [17, 18].

Os autores Von Halle e Goldberg [19] concordam que os casos de uso e as regras de negócio trabalham juntos. Mas, como eles representam considerações diferentes, deve-se escolher uma forma de separá-los. As regras de negócio como têm sua própria existência devem ter o seu próprio modelo, independentemente de como e onde essas são executadas, e se são automatizadas ou não. O principal benefício de separá-los é gerenciá-los de forma independente. Para tratar essa separação eles propõem associar os casos de uso a um repositório de regras de negócio. Com isso, os casos de uso ficam simplificados, pois, dentro destes, não estarão as regras de negócio.

\section{Rastreabilidade de requisitos}

O sucesso ou o fracasso de um projeto é determinado pela conformidade dos requisitos levantados com as características do sistema produzido, afirmam Leffingwell e Widrig [20]. Para descobrir os requisitos, é necessário escrevê-los, organizá-los e monitorá-los durante as mudanças. A engenharia de requisitos trata dessas atividades.

Wiegers [10] divide a engenharia de requisitos em duas áreas: desenvolvimento e gerenciamento de requisitos. O desenvolvimento de requisitos engloba as atividades de levantar, avaliar, documentar e validar os requisitos por meio dos processos de elicitação, análise, especificação e validação. O gerenciamento de requisitos envolve as atividades de controle de mudança, controle de versão, rastreabilidade do estado do requisito (proposto, aprovado, implementado, verificado, excluído e rejeitado) e rastreabilidade do requisito. Para gerenciar essas atividades de gerenciamento, deve haver um responsável que defina os mecanismos de armazenamento (como uma ferramenta de gerenciamento de requisitos) e os atributos dos requisitos. Além disso, o responsável deve gerenciar os estados dos requisitos e as atualizações de dados de rastreabilidade e gerar os relatórios de atividades de mudanças.

O gerenciamento de requisitos exige que as informações de rastreabilidade sejam registradas como, por exemplo, a ligação dos requisitos com as fontes dos requisitos e o projeto de sistema [21]. Nas palavras de Wiegers [10], a utilização de uma ferramenta auxilia no gerenciamento de requisitos, pois ajuda na realização das seguintes tarefas:

- manutenção do histórico das alterações de cada requisito para gerenciar as versões e as mudanças;

- armazenamento dos atributos dos requisitos como a data de criação do requisito e o número de versão;

- capacidade de consulta a subconjuntos de requisitos como, por exemplo, uma lista de requisitos derivados de uma regra de negócio para verificar as consequências de uma mudança na regra;

- ligação entre os requisitos para facilitar a análise da consequência de uma mudança, identificando os requisitos afetados;

- controle do estado de cada requisito durante o desenvolvimento para monitorar o estado do projeto;

- armazenamento dos requisitos em uma base de dados para facilitar o reuso e evitar a duplicação de requisitos.

De acordo Nuseibeh e Easterbrook [22], no centro do gerenciamento de requisitos está a rastreabilidade.

Todas essas reflexões podem ser encontradas como exigências em dois modelos de maturidade do desenvolvimento de software: o CMMI [23] e o MPS.BR [24]. Para o CMMI, a capacidade de gerenciar requisitos é uma exigência do nível 2 (o primeiro nível de maturidade). Um dos objetivos específicos da área de processo Gerência de Requisitos (GRE) é a manutenção da rastreabilidade bidirecional dos requisitos. Esse tipo de rastreabilidade pode ser definido de modo geral conforme Gotel e Finkelstein [25], como a capacidade de descrever e seguir a vida de um requisito, para frente e para trás, desde a sua origem, passando pela 
especificação, desenvolvimento até a implantação e uso, e durante o refinamento e a iteração das fases. Para o MPS.BR, no nível G (o primeiro passo na direção do amadurecimento dos processos de desenvolvimento de software), exige-se a implantação do processo de Gerência de Requisitos (GRE), sendo a rastreabilidade bidirecional um de seus resultados esperados.

Em termos gerais, a rastreabilidade é usada para diferentes finalidades. Para o gerente, com o intuito de mostrar que os requisitos acordados foram satisfeitos. Para o desenvolvedor, para acompanhar a evolução dos requisitos e verificar se esses foram atendidos pelo sistema. Para as equipes de projeto e de manutenção, a fim de verificar o que deve ser alterado para atender às mudanças, independentemente do conhecimento do desenvolvedor [26].

Requisitos não podem ser gerenciados sem rastreabilidade. Em Kotonya e Sommerville [21], um requisito é rastreável quando se identifica quem o solicitou (a fonte), por que da sua existência (razão), quais os outros requisitos relacionados a esse (dependência entre requisitos) e como esse relaciona-se com as outras informações de projeto, de implementação e de documentação do sistema. A informação de rastreabilidade é usada para encontrar os requisitos afetados por uma mudança.

Segundo Aizenbud-Reshef et al. [26], como os requisitos normalmente são escritos de maneira informal, exige-se que as pessoas os entendam e os validem manualmente, tornando inviável manter as informações de rastreabilidade atualizadas. Além disso, para possibilitar a rastreabilidade, cada requisito deve ser único e rotulado para que se possa consultá-lo em todo o sistema [10].

Uma classificação geral da rastreabilidade de requisitos é dividida em: pré e pós-rastreabilidade. A prérastreabilidade (para trás) documenta a origem dos requisitos e a pós-rastreabilidade (para frente) associa os requisitos ao projeto e a implementação do sistema [27].

A rastreabilidade de requisitos teve início nos anos de 1970 para responder às questões sobre a necessidade de determinado requisito, sua forma de implementação e as consequências de uma mudança. $O$ primeiro método usado para o rastreamento foi a referência cruzada [26]. Desde então, diferentes técnicas têm sido utilizadas para representá-la.

Ramesh e Jarke [1] realizaram um estudo que divide os participantes em dois grupos de usuário: baixo e alto nível. Os usuários de baixo nível são inexperientes e consideram a rastreabilidade uma obrigação vinda de uma ordem superior ou para atender as normas. Os usuários de alto nível são experientes e consideram a rastreabilidade um meio de satisfazer o cliente e de ter um acompanhamento durante todo o desenvolvimento.

O usuário de alto nível preocupa-se em documentar as razões das decisões que podem ser usadas em desenvolvimentos futuros para evitar o retrabalho. Por essa característica, o modelo para usuário de alto nível é utilizado para estruturar a rastreabilidade dos requisitos e as razões das decisões no repositório proposto. Letelier [28] também destaca a importância em documentar a razão das decisões e os responsáveis pela criação/modificação das especificações.

\section{Repositório de requisitos funcionais de software e regras de negócio}

Um repositório de requisitos de software possibilita armazenar, compartilhar e gerenciar os requisitos e as relações entre esses. Essas relações permitem encontrar os requisitos afetados por uma mudança. O repositório também facilita a comunicação da equipe por meio do compartilhamento das informações, o reuso dos requisitos em outros sistemas e preserva a memória organizacional, evitando que alterações na equipe levem à perda do conhecimento [29,30]. Em relação às regras de negócio, como essas são definidas e refinadas durante o ciclo de vida do sistema, é importante dispor-se de um repositório para gerenciá-las, mas esse repositório não deve conter somente as definições das regras, mas também os seus relacionamentos [18].

Pelos benefícios citados, o presente trabalho adota o repositório para armazenamento dos requisitos funcionais de software, das regras de negócio e das decisões de negócio. O modelo do repositório proposto resulta da contribuição de vários modelos. O modelo de caso de uso, sugerido pelo Rational Unified Process [14], é utilizado para representar os requisitos funcionais de software, o modelo do repositório elaborado por Ciavatta [31] para tratar as regras de negócio/decisões de negócio e a associação do caso de uso ao repositório de regras de negócio proposto por Von Halle e Goldberg [19]. 
Para estruturar a rastreabilidade dos requisitos é adotado o modelo proposto por Ramesh e Jarke [1] e o metamodelo elaborado por Letelier [28], que destacam a importância em documentar as razões das decisões de negócio. A Figura 1 apresenta a formação do repositório proposto.

Figura 1: Repositório

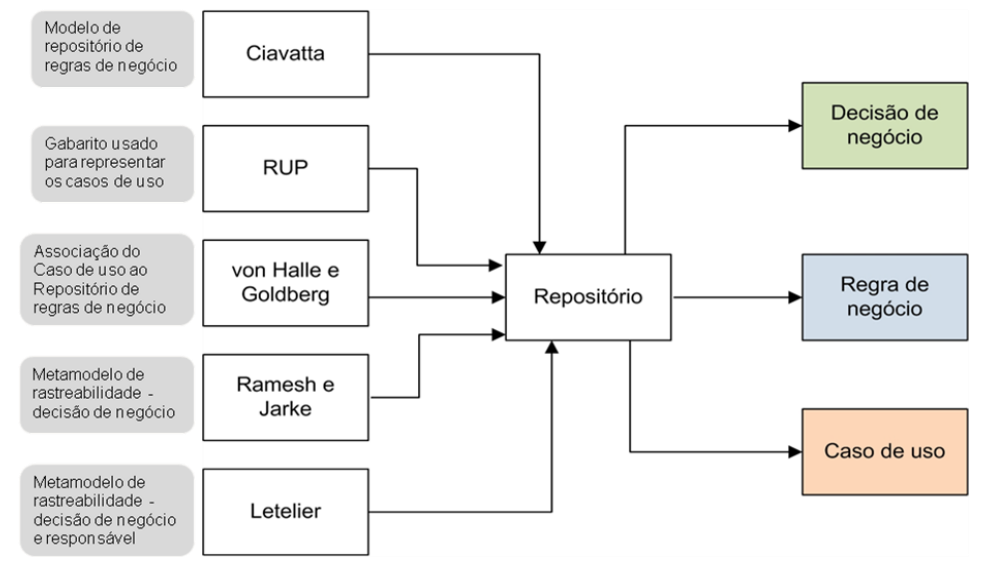

O repositório para o rastreamento de requisitos funcionais derivados de regras de negócio, proposto neste estudo, baseia-se em dois modelos: conceitual e de rastreabilidade. O modelo conceitual é composto pelos elementos e pelas associações das decisões de negócio, das regras de negócio e dos casos de uso. O modelo de rastreabilidade é formado pelos elementos e pelas associações que compõem a rastreabilidade dos elementos do modelo conceitual, definidos como itens rastreáveis. Os modelos apresentam a regra de negócio como a ligação entre as decisões de negócio e os casos de uso, possibilitando o rastreamento dos requisitos funcionais de software e sua origem nos negócios. A notação adotada para representar o modelo conceitual e o modelo de rastreabilidade é o diagrama de classes da Unified Modeling Language (UML), cujo objetivo é descrever os objetos do sistema e os relacionamentos entre esses.

\subsection{Modelo de rastreabilidade}

Confome Ramesh e Jarke [1], normalmente somente as alternativas escolhidas são documentadas, as descartadas não são registradas. Com a evolução dos requisitos, a disponibilidade dessas informações ajuda a evitar o retrabalho, pois o histórico pode ser usado em manutenções e desenvolvimentos futuros. Além disso, possibilita acompanhar o estado do desenvolvimento, as mudanças feitas e os motivos.

Para Letelier [28], registrar a razão contribui para melhorar a aceitação do sistema pelos envolvidos e aperfeiçoar o gerenciamento de mudanças, evitando estudar novamente as considerações já excluídas.

O modelo de rastreabilidade proposto é formado pelo item rastreável e pelo decisor, como proposto por Letelier [28]. O decisor representa os responsáveis por criar ou modificar os itens rastreáveis. O item rastreável é uma generalização da decisão de negócio, da regra de negócio e do caso de uso (representando o requisito funcional de software). Uma decisão é a razão dos itens rastreáveis. A regra de negócio é a ligação da decisão de negócio com o caso de uso. Uma decisão de negócio pode afetar zero ou mais regras de negócio. Uma regra de negócio pode afetar zero ou mais casos de uso.

Para facilitar a descrição, a maioria dos componentes conceituais do modelo de rastreabilidade foi agrupada em submodelos: submodelo conceitual decisão de negócio, submodelo conceitual regra de negócio e submodelo conceitual caso de uso. A Figura 2 apresenta o diagrama de classes (UML) do modelo de rastreabilidade de requisitos de software proposto, destacando apenas seus elementos centrais por questões de legibilidade.

- Decisão de negócio constitui o elemento central do submodelo conceitual decisão de negócio.

- Regra de negócio constitui o elemento central do submodelo conceitual regra de negócio.

- Caso de uso constitui o elemento central do submodelo conceitual caso de uso. 
Figura 2: Modelo de rastreabilidade de requisitos de software

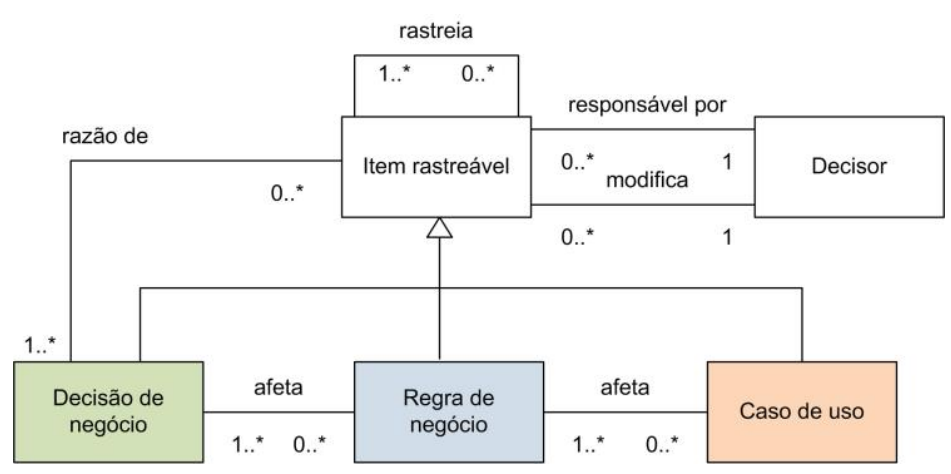

\subsection{Submodelos conceituais}

O submodelo conceitual decisão de negócio é estruturado com a razão das decisões de negócio e tem como objetivo avaliar e definir a direção da empresa por meio do impacto potencial das decisões, a qual está representada no submodelo pela decisão de negócio. A decisão de negócio apresenta alternativas. As alternativas são partes das premissas consideradas e dos argumentos elaborados durante o processo decisório. As decisões de negócio são tomadas pelo decisor responsável com base nas alternativas, sendo que a alternativa escolhida é denominada "é escolhida". As avaliações verificam as premissas e validam os argumentos. As decisões de negócio podem afetar as regras de negócio. A Figura 3 apresenta o diagrama de classes (UML) que representa a descrição do submodelo conceitual decisão de negócio.

Figura 3: Submodelo conceitual decisão de negócio

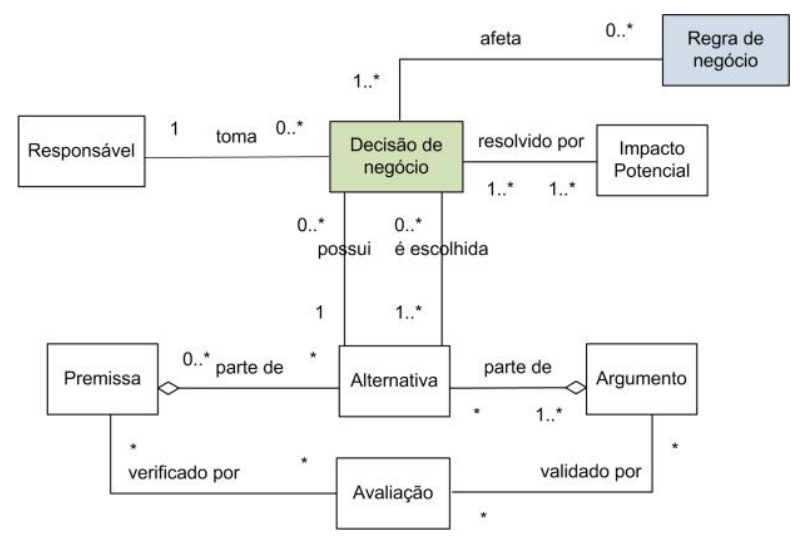

O submodelo conceitual regra de negócio apresenta a estrutura de uma regra de negócio, os tipos de controle que podem ser exercidos e seus estágios de evolução.

As regras de negócio são formadas por fatos e os fatos são compostos por termos [3]. As regras podem ser classificadas em internas ou externas. As externas são definidas por órgãos externos, tais como órgãos reguladores e governamentais. As internas são definidas pela empresa e apresenta um decisor responsável, seja um funcionário ou um departamento. Toda regra de negócio é validada por um tipo de regra. Cada tipo de regra tem uma família. Cada família executa um tipo específico de teste que pode ser aplicado no sistema de informação. Portanto, o mesmo teste não pode ser aplicado em famílias diferentes [32]. Uma regra de negócio exerce um tipo de controle, chamado classificação funcional [33]. Os estágios de evolução de uma regra de negócio são representados pelo Estado, o qual é usado para registrar as etapas de uma regra de negócio, permitindo obter o histórico de mudanças de determinada regra. Os estados podem ser configurados de acordo com a necessidade do projeto. As decisões de negócio podem afetar as regras de negócio e as regras de negócio 
podem afetar os casos de uso. A Figura 4 apresenta o diagrama de classes (UML) que representa a descrição do submodelo conceitual regra de negócio.

Figura 4: Submodelo conceitual regra de negócio

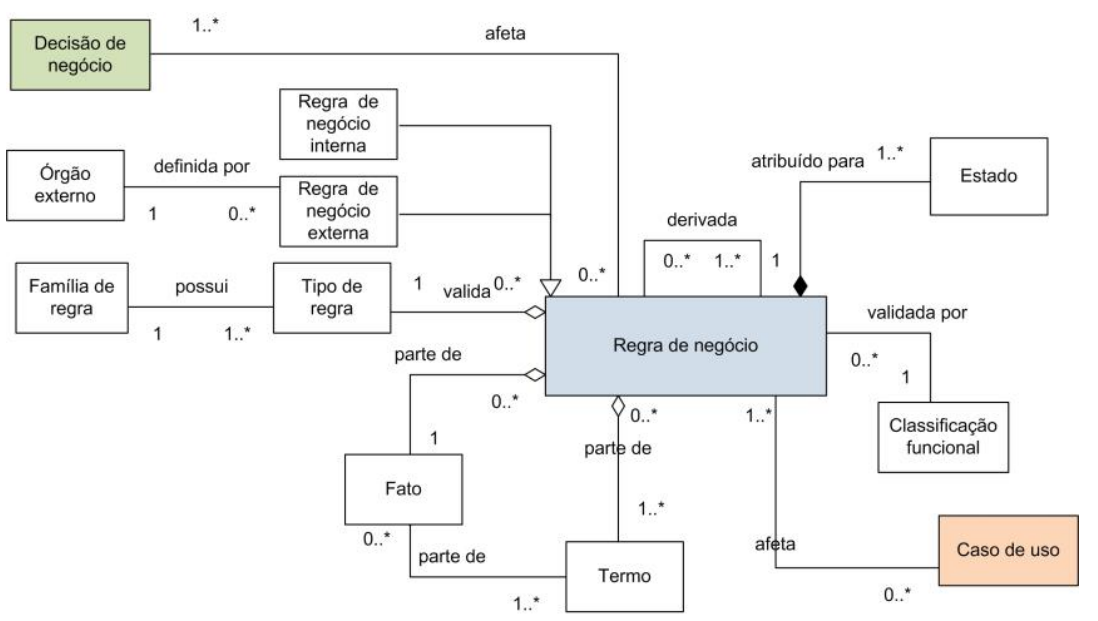

O submodelo conceitual caso de uso apresenta a estrutura de um caso de uso e seus estágios de evolução. A estrutura de caso de uso adotada para este trabalho é baseada no gabarito RUP [14]. Um caso de uso é formado por no mínimo um ator e um fluxo básico. A precondição e pós-condição são opcionais. Um caso de uso pode ter zero ou mais fluxos alternativos, nos quais são descritos os cenários alternativos relacionados ao fluxo básico. O fluxo básico pode ter zero ou mais extensões identificadas por pontos de extensão.

Os requisitos especiais não são usados, pois o trabalho aqui proposto é restrito aos requisitos funcionais de software. As regras de negócio podem afetar os casos de uso. Por esse motivo, como proposto por Von Halle e Goldberg [19] deve haver uma associação dos casos de uso com as regras de negócio. Os estágios de evolução de um caso de uso são representados pelo estágio. O estágio é usado para registrar as etapas de um caso de uso, permitindo obter o histórico de mudanças de determinado caso de uso. Os estágios podem ser configurados de acordo com a necessidade do projeto. Os casos de uso são definidos pela área de sistema da empresa e apresentam um decisor responsável por documentá-los, chamado desenvolvedor. A Figura 5 apresenta o diagrama de classes (UML) que representa a descrição do submodelo conceitual caso de uso.

Figura 5: Submodelo conceitual caso de uso

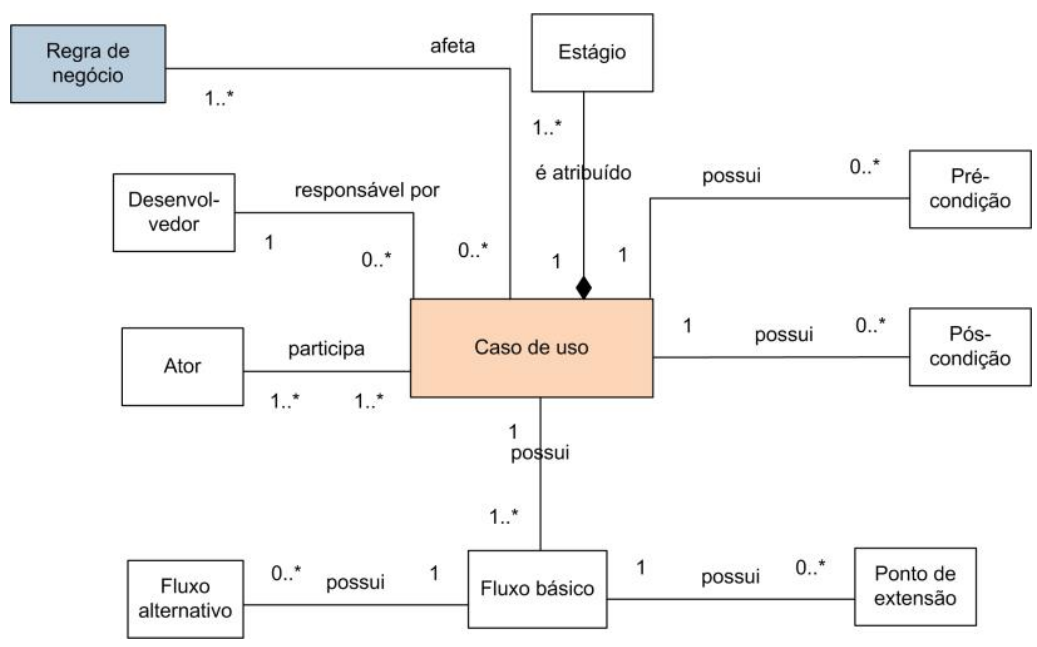




\section{Exemplo de aplicação do repositório}

A aplicação do exemplo é feita por meio de um protótipo, chamado decisão de negócio, regra de negócio e caso de uso (DRC), desenvolvido na ferramenta Microsoft Visual Studio.Net e com o gerenciador de banco de dados Microsoft SQL Server, com o objetivo de implementar o repositório proposto. O protótipo possibilita:

- cadastrar as informações da decisão de negócio;

- cadastrar as características da regra de negócio e armazenar o histórico de alterações;

- cadastrar as características do caso de uso e armazenar o histórico de alterações;

- cadastrar a rastreabilidade da decisão de negócio e da regra de negócio;

- cadastrar a rastreabilidade da regra de negócio e do caso de uso;

- consultar, por meio de filtros de pesquisa, as decisões de negócio;

- consultar, por meio de filtros de pesquisa, o histórico e o detalhe das regras de negócio para acompanhar sua evolução;

- consultar, por meio de filtros de pesquisa, o histórico e o detalhe dos casos de uso para acompanhar sua evolução;

- consultar, por meio de filtros de pesquisa, as decisões de negócio relacionadas às regras de negócio, para analisar as consequências de uma mudança;

- consultar, por meio de filtros de pesquisa, as regras de negócio relacionadas aos casos de uso para analisar as consequências de uma mudança.

O modelo de rastreabilidade, implementado no protótipo, é composto pela especificação dos itens rastreáveis: submodelos conceituais decisão de negócio, regra de negócio e caso de uso, do modelo de rastreabilidade descrito na seção 4, e pela gerência de rastreabilidade. A especificação é formada pela inclusão e atualização do item rastreável. A gerência é formada pela especificação e consulta da rastreabilidade. A Figura 6 ilustra o modelo de rastreabilidade do protótipo.

Figura 6: Modelo do protótipo

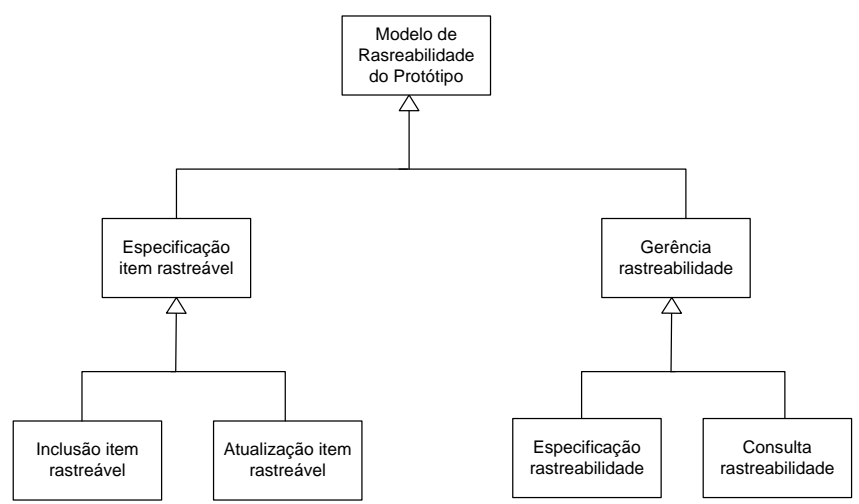

A Tabela 1 apresenta a relação entre os elementos do modelo de rastreabilidade (Figura 2) com o modelo do protótipo (Figura 6) e os componentes do protótipo. A Figura 7 ilustra o mapeamento apresentado na Tabela 1 .

Tabela 1: Modelo de rastreabilidade e do protótipo e componente do protótipo

\begin{tabular}{lll}
\hline \multicolumn{1}{c}{ Modelo de rastreabilidade } & \multicolumn{1}{c}{ Modelo do protótipo } & Componente do protótipo \\
\hline Decisão de negócio & Especificação item rastreável & Decisão de negócio \\
Regra de negócio & Especificação item rastreável & Regra de negócio \\
Caso de uso & Especificação item rastreável & Caso de uso \\
Relacionamento (“afeta”) & Especificação rastreabilidade & Rastreabilidade \\
& Consulta rastreabilidade & Consulta \\
\hline
\end{tabular}


Figura 7: Mapeamento do protótipo

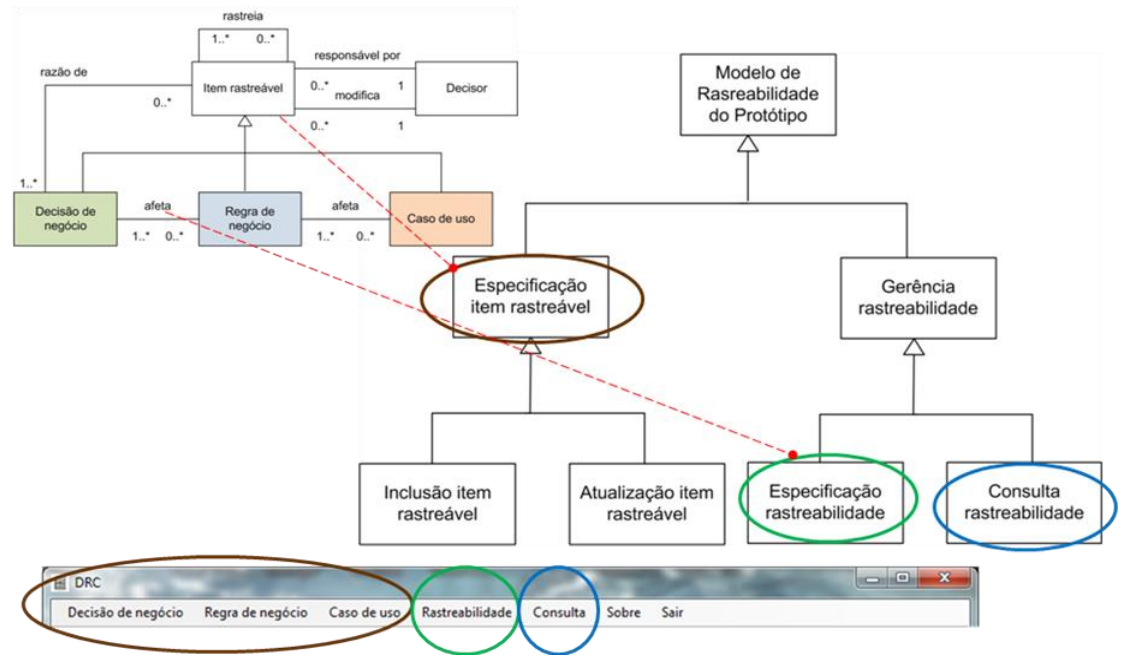

A seguir são apresentados os componentes do protótipo em uma sequência que simula a especificação dos itens rastreáveis e a gerência de rastreabilidade, para que se possa observar o uso do repositório proposto. As telas apresentam dados extraídos de um estudo de caso, descrito pela BRG [3], de uma empresa fictícia de aluguel de carros, chamada EU-Rent. O componente do protótipo decisão de negócio tem a finalidade de possibilitar a identificação das informações relacionadas à decisão de negócio tomada pela área de negócio da empresa, conforme apresentado na Figura 8.

Figura 8: Tela decisão de negócio

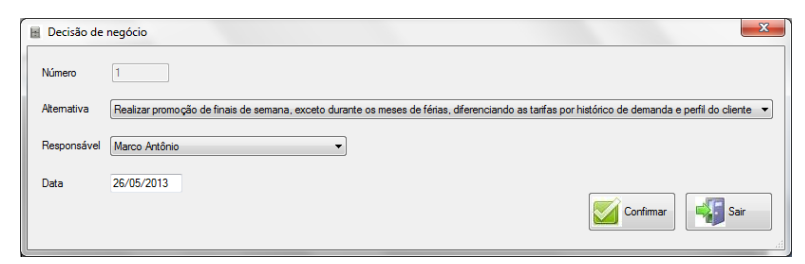

No componente do protótipo regra de negócio definem-se as características de uma regra de negócio, e possibilitando o acompanhamento da evolução da regra de negócio pela área de negócio da empresa, conforme apresentado na Figura 9. Uma nova versão é gerada a cada alteração na tela.

Figura 9: Tela regra de negócio

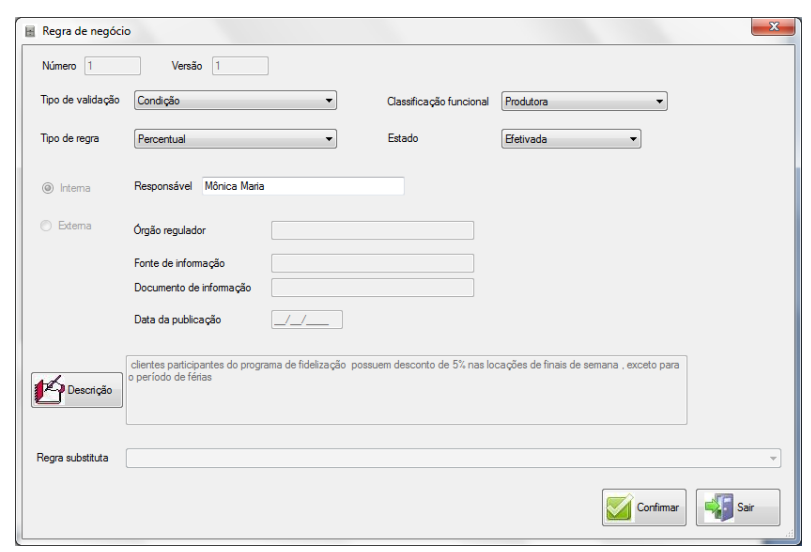

No componente do protótipo caso de uso definem-se as características de um caso de uso, possibilitando o acompanhamento da evolução do caso do uso pela área de desenvolvimento de sistema da empresa, conforme apresentado na Figura 10. Uma nova versão do caso de uso é gerada a cada alteração na tela. 
Figura 10: Tela caso de uso

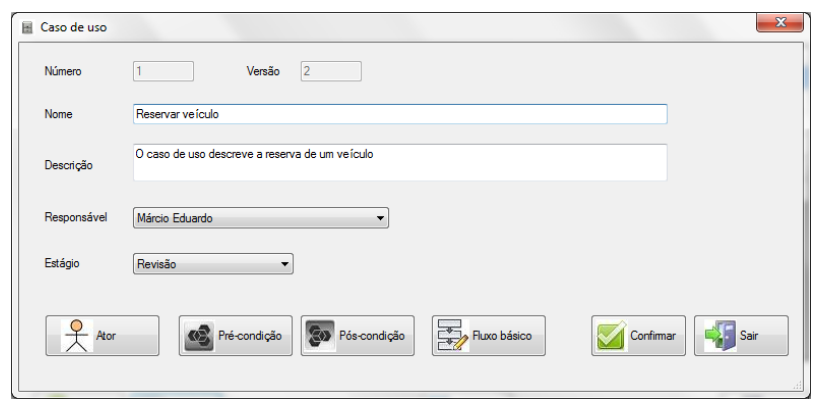

No componente do protótipo rastreabilidade define-se a rastreabilidade dos itens especificados: decisão de negócio, regra de negócio e caso de uso, no escopo da gerência de rastreabilidade. As telas de rastreabilidade são apresentadas a seguir. A Figura 11 mostra a tela de especificação da rastreabilidade da decisão de negócio e da regra de negócio. A Figura 12 ilustra a tela de especificação da rastreabilidade da regra de negócio e do caso de uso.

Figura 11: Tela rastreabilidade decisão de negócio e regra de negócio

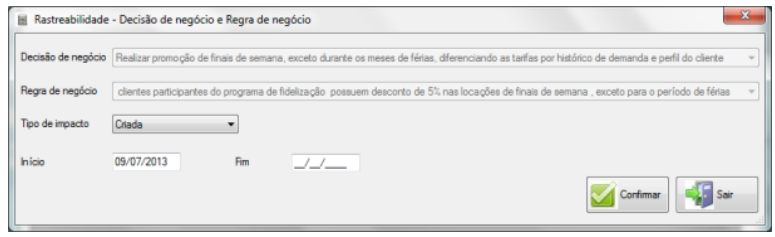

Figura 12: Tela rastreabilidade regra de negócio e caso de uso

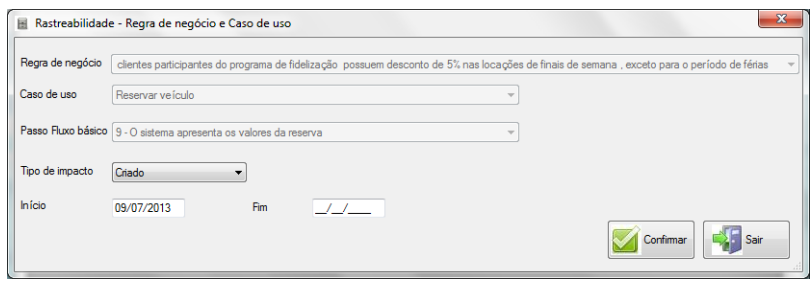

Após a especificação dos itens rastreáveis e da rastreabilidade, pode-se consultar o histórico desses itens e suas relações por meio das telas de consultas. A finalidade das telas de consulta é possibilitar o gerenciamento das decisões de negócio tomadas pela empresa, o acompanhamento da evolução das regras de negócio, o acompanhamento da evolução dos casos de uso e o rastreamento dos requisitos e sua origem nos negócios. A Figura 13 apresenta a tela de consulta da associação da regra de negócio e do caso de uso.

Figura 13: Tela consulta regra de negócio e caso de uso

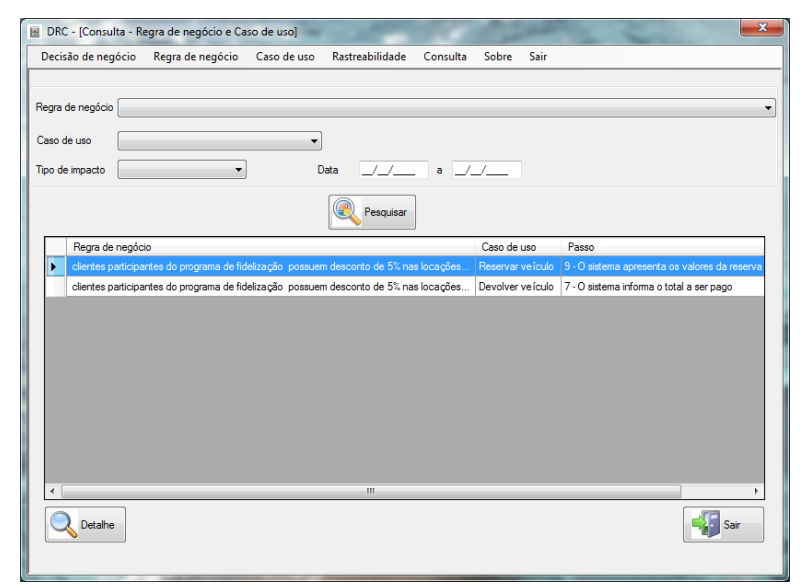




\section{Avaliação preliminar da aplicação do repositório}

Para avaliar preliminarmente a aplicabilidade do repositório para o rastreamento de requisitos funcionais foram analisados critérios baseados nas tarefas sugeridas por Wiegers [10] para uma ferramenta de gerenciamento de requisitos. A Tabela 2 mostra essa avaliação, que é formada pelos seguintes itens:

- critério: descreve o critério usado para avaliação;

- protótipo: descreve a funcionalidade do protótipo que atende ao critério proposto;

- resultado: mostra o resultado da avaliação, se o protótipo "atende" ou "não atende" ao critério proposto.

Tabela 2: Avaliação do repositório: requisito

\begin{tabular}{l} 
Critério \\
\hline Manutenção do histórico das alterações de cada \\
requisito para gerenciar as versões e as mudanças. \\
Armazenamento dos atributos dos requisitos como \\
a data de criação do requisito e o número de versão. \\
Capacidade de consulta a subconjuntos de \\
requisitos como, por exemplo, uma lista de \\
requisitos derivados de uma regra de negócio para \\
verificar as consequências de uma mudança na \\
regra. \\
Ligação entre os requisitos para facilitar a análise \\
da consequência de uma mudança, identificando os \\
requisitos afetados.
\end{tabular}

Controle do estado de cada requisito durante o desenvolvimento para monitorar o estado do projeto.

Armazenamento dos requisitos em uma base de dados para facilitar o reuso e evitar a duplicação de requisitos.

\section{Protótipo}

Uma nova versão é gerada a cada alteração na
tela de caso de uso, armazenando o histórico do caso de uso.

A tela de caso de uso armazena as características do caso de uso como a data da criação e o número da versão.

Associação da regra de negócio e caso de uso que possibilita a consulta aos casos de uso associados às regras de negócio.

Inclusão de um caso de uso existente ao caso de uso base.

Associa um caso de uso de extensão ao caso de uso base.

A tela de consulta ao caso de uso possibilita consulta aos estados dos casos de uso.

Dados armazenados na base de dados DRC.
Atende

Resultado

Atende

Atende

Atende

Atende

Atende

O protótipo desenvolvido armazena os atributos dos requisitos, os responsáveis, as versões, os relacionamentos com outros requisitos e os relacionamentos com as regras de negócio na base de dados. As telas de consulta permitem que a área de desenvolvimento de sistemas consulte o histórico dos requisitos e os responsáveis, os relacionamentos para análise das consequências de uma mudança e acompanhe os estágios de evolução dos requisitos para monitorar o estado do projeto. Considerando essas características, pode-se concluir que o protótipo desenvolvido atende aos critérios propostos no que diz respeito aos requisitos funcionais representados em casos de uso.

Além de contribuir para documentar a história dos requisitos, o protótipo possibilita registrar as regras de negócio criadas, documentando, também a origem desses requisitos nos negócios. A Tabela 3 mostra a avaliação do repositório no que se refere às regras de negócio, aplicando os critérios usados nos requisitos adaptados para as regras de negócio.

O protótipo também armazena os atributos das regras de negócio, os responsáveis, as versões e os relacionamentos com as decisões de negócio na base de dados. Para complementar o repositório, as informações relacionadas às decisões de negócio também são armazenadas na base de dados. As telas de consulta possibilitam que a área de negócio da empresa consulte as decisões tomadas, o histórico das regras de negócio e os responsáveis, os relacionamentos para análise das consequências de uma mudança e acompanhe os estados de evolução das regras. Considerando essas características, pode-se concluir que o protótipo desenvolvido atende aos critérios propostos no que diz respeito às regras de negócio. 
Tabela 3: Avaliação do repositório: regra de negócio

\begin{tabular}{|c|c|c|}
\hline Critério & Protótipo & Resultado \\
\hline $\begin{array}{l}\text { Manutenção do histórico das alterações de cada } \\
\text { regra de negócio para gerenciar as versões e as } \\
\text { mudanças. }\end{array}$ & $\begin{array}{l}\text { Uma nova versão é gerada a cada alteração na } \\
\text { tela de regra de negócio, armazenando o histórico } \\
\text { da regra de negócio. }\end{array}$ & Atende \\
\hline $\begin{array}{l}\text { Armazenamento dos atributos das regras de } \\
\text { negócio como a data de criação da regra e o } \\
\text { número de versão. }\end{array}$ & $\begin{array}{l}\text { A tela de regra de negócio armazena as } \\
\text { características da regra de negócio como a data da } \\
\text { criação e o número da versão. }\end{array}$ & Atende \\
\hline $\begin{array}{l}\text { Capacidade de consulta a subconjuntos de regras } \\
\text { de negócio como, por exemplo, uma lista de regras } \\
\text { de negócio derivadas de uma decisão de negócio } \\
\text { para verificar as consequências de uma mudança na } \\
\text { decisão. }\end{array}$ & $\begin{array}{l}\text { Associação da decisão de negócio e regra de } \\
\text { negócio, que possibilita a consulta às regras de } \\
\text { negócio associados às decisões de negócio. }\end{array}$ & Atende \\
\hline Controle do estado de cada regra de negócio. & $\begin{array}{l}\text { A tela de consulta à regra de negócio possibilita } \\
\text { consulta aos estados das regras de negócio. }\end{array}$ & Atende \\
\hline $\begin{array}{l}\text { Armazenamento das regras de negócio em uma } \\
\text { base de dados para facilitar o reuso e evitar a } \\
\text { duplicação de regras. }\end{array}$ & Dados armazenados na base de dados DRC. & Atende \\
\hline
\end{tabular}

\section{Conclusão}

As regras de negócio e os sistemas de informação são afetados pelas mudanças nos negócios de uma empresa. Para que os sistemas sejam consistentes com os negócios deve haver uma documentação que possibilite determinar as implicações das mudanças, permitindo rastrear separadamente as regras de negócio e os requisitos de software e as relações entre esses. Para documentar as regras de negócio e os requisitos de software separados, o presente trabalho propôs um repositório formado pelas informações relacionadas às decisões de negócio, pelas características das regras de negócio e dos requisitos funcionais de software que possibilita rastreá-los.

Para representar os requisitos funcionais de software foi utilizado um modelo de caso de uso, tendo sua estrutura baseada no gabarito RUP [14]. Para tratar as regras de negócio e as decisões de negócio foi usado como base o repositório elaborado por Ciavatta [31]. A rastreabilidade dos requisitos foi baseada no modelo conceitual proposto por Ramesh e Jarke [1] e no metamodelo elaborado por Letelier [28], que destacam a importância em documentar a razão das decisões de negócio. O repositório baseia-se em dois modelos: de rastreabilidade e conceitual. O modelo de rastreabilidade é formado pelo item rastreável e pelo decisor. O item rastreável representa a decisão de negócio, a regra de negócio e o caso de uso. O decisor representa o responsável por criar ou modificar o item rastreável. O modelo conceitual apresenta os elementos e as associações dos itens rastreáveis: decisão de negócio, regra de negócio e caso de uso.

Para ilustrar a aplicabilidade do repositório proposto foi apresentado um protótipo que permitiu simular a especificação/consulta: das decisões de negócio, das regras de negócio, dos casos de uso, das associações das decisões de negócio/regras de negócio e das associações das regras de negócio/casos de uso.

Baseado nos resultados preliminares apresentados e considerando o caráter conceitual do presente trabalho, conclui-se que o repositório proposto permite atender as principais necessidades das áreas de negócio e de sistemas da empresa, no que diz respeito à rastreabilidade dos requisitos, visto que proporciona consultas aos dados especificados por cada área, possibilitando atribuir responsabilidades e efetuar avaliações de modificações futuras e de desenvolvimento de novos sistemas. No entanto, somente uma avaliação em um ambiente real poderá fazer um julgamento mais preciso do repositório proposto. Assim, o principal trabalho futuro previsto é a realização de um estudo de caso que considere as decisões de negócio reais de uma empresa na especificação das regras de negócio e dos requisitos funcionais de software. A definição do protocolo do estudo de caso tomará como base os critérios preliminares apresentados neste artigo. 


\section{Referências}

[1] RAMESH, B.; JARKE, M. Towards reference models for requirements traceability. IEEE Transactions on Software Engineering, v.27, n.1, p.58-93, Jan. 2001.

[2] ROSS, R.G. Why Rulebook Management? Because Software Requirements and Business Rules Simply Aren't the Same! Business Rules Journal, v.12, n.1, Jan. 2011. Disponível em: <http://www.BRCommunity.com/a2011/b572.html>. Acesso em: 28 ago. 2011.

[3] BUSINESS RULE GROUP. Defining Business Rules: what are they really?, v. 1.3, July 2000. Disponível em: <http://www.businessrulesgroup.org/first_paper/BRG-whatisBR_3ed.pdf>. Acesso em: 14 jun. 2011.

[4] MATIGNON, C. capturing business rule you don’t know. Business Rules Journal, v.12, n.4, Apr. 2011. Disponível em: <http://www.BRCommunity.com/a2011/b590.html>. Acesso em: 7 set. 2011.

[5] BAJEC, M.; KRISPER, M. A methodology and tool support for managing business rules in organizations. Information Systems Journal, v.30, n.6, p.423-443, Sept. 2005.

[6] STEINKE, G.; NICKOLETTE, C. Business rule as the basic of an organization's information systems. Industrial Management \& Data Systems, v.103, n.1, p.52-63, 2003.

[7] NIJPELS, R. Business Rules in Requirements Analysis. Business Rules Journal, v.6, n.12, Dec. 2005. Disponível em: <http://www.brcommunity.com/b259.php>. Acesso em: 25 fev. 2012.

[8] SWEBOK - Guide to the software engineering body of knowledge, 2004. Disponível em: <http://www.computer.org/portal/web/swebok/htmlformat>. Acesso em: 14 jul. 2012.

[9] GOTTESDIENER, E. Top ten ways project teams misuse use cases: and how to correct them, 2002. Disponível

em: <http://www.ibm.com/developerworks/rational/library/content/RationalEdge/jun02/MisuseUseCasesJun02.pdf>. Acesso em: 7 mar. 2012.

[10] WIEGERS, K. E. Software requirements, Second Edition. Microsoft Press, 2003. 516 p.

[11] PRESSMAN, R. S. Engenharia de software. Mc Graw Hill, 2006. 720 p.

[12] JACOBSON, I. Use cases: yesterday, today, and tomorrow. Software and Systems Modeling, local de publicação, v.3, n.3, p. 210-220, mês abreviado. 2004.

[13] FOWLER, M. UML Essencial. Uma breve guia para a linguagem-padrão de modelagem de objetos. 3. ed. Bookman, 2005. 160 p.

[14] RATIONAL UNIFIED PROCESS. Especificação de caso de uso v. 1, 2003. Disponível em:

<http://www.wthreex.com/rup/portugues/webtmpl/templates/req/rup_ucspec.htm/>. Acesso em: 15 nov. 2012.

[15] COCKBURN, A. Escrevendo casos de uso eficazes. Bookman, 2005.

[16] KULAK, D.; GUINEY, E. Use cases: requirements in context. 2. ed. Addison- Wesley, 2003.

[17] GOTTESDIENER, E. Capturing business rules. Software Development Magazine, v.7, n.12, Dec. 1999.

[18] MORGAN, T. Business rules and information systems: aligning it with business goals. Addison-Wesley, 2002.

[19] VON HALLE, B.; GOLDBERG, L. Use cases and business rules: can they work together?, 2010. Disponível

em:<http://www.modernanalyst.com/Resources/Articles/tabid/115/articleType/ArticleView/articleId/1442/U se-Cases-and-Business-Rules-Can-They-Work-Together.aspx>. Acesso em: 7 jul. 2012.

[20] LEFFINGWELL, D.; WIDRIG, D. Introduction to requirements management. In: Managing Software Requirements: a use case approach. 2. ed. Addison-Wesley, 2003, p. 15-22.

[21] KOTONYA, G.; SOMMERVILLE I. Requirements engineering: processes and techniques. Wiley, 1998. 
[22] NUSEIBEH, B.; EASTERBROOK, S. Requirements engineering: a roadmap proceedings of the conference on the future of software engineering, 2000, p. 35-46.

[23] SOFTWARE ENGINEERING INSTITUTE. CMMI for Development, Version 1.3, Nov. 2010.

[24] MPS.BR - Melhoria de Processo de Software Brasileiro, Guia Geral MPS de Software, Dez. 2012.

[25] GOTEL, O. C. Z.; FINKELSTEIN, A. C. W. An analysis of the requirements traceability problem. PROCEEDINGS OF THE FIRST INTERNATIONAL CONFERENCE ON REQUIREMENTS ENGINEERING, 1994, p. 94-101, Apr. 1994.

[26] AIZENBUD-RESHEF, N. et al. Model traceability. IBM Systems Journal, local de publicação, v. 45, n. 3, p. 515-526, July 2006.

[27] POHL, K. PRO-ART: Enabling requirements pre-traceability. PROCEEDINGS OF THE SECOND INTERNATIONAL CONFERENCE ON REQUIREMENTS ENGINEERING, 1996, p. 76-84, Apr. 1996.

[28] LETELIER, P. A framework for requirements traceability in uml-based projects. Proceeding of 1st International Workshop on Traceability in Emerging Forms of Software Engineering, p.32-41, 2002.

[29] BLAHA, M.; LAPLANT, D.; MARVAK, E. Requirements for repository software. PROCEEDINGS OF THE FIFTH WORKING CONFERENCE ON REVERSE ENGINEERING, 1998, p. 164-173, Oct. 1998.

[30] KAPOCIUS, K.; BUTLERIS, R. Repository for business rules based is requirements. Journal Informatica, v.17, n.4, p. 503-518, Dec. 2006.

[31] CIAVATTA, D. Um modelo de repositório para o Rastreamento de Regras de Negócio em Aplicações de Sistemas de Informação. São Paulo, 2009. Dissertação (Mestrado profissional) - Coordenadoria de Ensino Tecnológico, Instituto de Pesquisas Tecnológicas do Estado de São Paulo, São Paulo, 2009.

[32] ROSS, R.G. The business rule book: classifying, defining and modeling rules. 2. ed. Business Rule Solutions Inc, 1997.

[33] ROSS, R.G. Principles of the business rule approach. Addison-Wesley, 2003. 352 p. 